MÁRCIA HELENA YAMAMOTO SATO

ANÁLISE DE ESTRUTURAS EM TAIPA PILÃO

São Paulo 
MÁRCIA HELENA YAMAMOTO SATO

\section{ANÁLISE DE ESTRUTURAS EM TAIPA DE PILÃO}

Dissertação apresentada à Escola Politécnica da Universidade de São Paulo para obtenção do título de Mestre em Engenharia 


\section{ANÁLISE DE ESTRUTURAS EM TAIPA DE PILÃO}

Dissertação apresentada à Escola Politécnica da Universidade de São Paulo para obtenção do título de Mestre em Engenharia

Área de Concentração:

Engenharia de Estrutura e

Fundação

Orientador: Prof ${ }^{\circ}$. Dr.

Reyolando Manoel Lopes Rebello da Fonseca Brasil

São Paulo 
Este exemplar foi revisado e alterado em relação à versão original, sob responsabilidade única do autor e com a anuência de seu orientador.

São Paulo, 03 de agosto de 2011.

Assinatura do autor:

Assinatura do orientador:

\section{Sato, Márcia Helena Yamamoto}

Análise de estruturas em taipa de pilão / M.H.Y. Sato. -- ed. rev. -- São Paulo, 2011.

$87 \mathrm{p}$.

Dissertação (Mestrado) - Escola Politécnica da Universidade de São Paulo. Departamento de Engenharia de Estruturas e Geotécnica.

1. Estruturas (Análise) 2. Taipa de pilão 3. Construção em terra 4. Técnicas tradicionais

I. Universidade de São Paulo. Escola Politécnica. Departamento de Engenharia de Estruturas e Geotécnica II. t. 
Ao meu marido, Alexandre, pelo incentivo a nunca desistir, mesmo tendo uma derrota, mas nunca cair, jamais. 


\section{AGRADECIMENTOS}

A DEUS, que me deu "vida e inteligência", e que me dá força para continuar a caminhada em busca do conhecimento, porque sabemos que desta vida levamos somente o “CONHECIMENTO” e deixamos o que se diz "RESPEITO”.

Meus sinceros agradecimentos a todos os que colaboraram para a realização deste trabalho, aqueles que estiveram ao meu lado acompanhando os avanços ou solidarizando-se nos impasses.

Agradeço aos meus pais, Cleuza e Tamotu, pelo apoio em todos os momentos e em especialmente aos meus queridos filhos, Natália Lumi e Alexandre Yudi, pela ajuda, incentivo e por sempre achar que a mamãe conseguiria chegar até aqui.

A minha prima (de coração) Margarete Miyagima, grande incentivadora e colaboradora, para que isso fosse possível.

Aos meus "amigos de verdade" eng ${ }^{\circ}$. Fernando Gonçalves, eng ${ }^{\circ}$. Leonardo Lago, eng ${ }^{\circ}$. Paulo Nigro e outros tantos colegas de profissão que pude conquistar durante esse mestrado.

Ao Prof $^{\circ}$. Dr. Reyolando M. L. R. da F. Brasil, meu orientador, por aceitar o tema escolhido e indicar sempre a direção mais adequada a seguir.

Aos Prof ${ }^{\circ}$. Dr. Osvaldo S. Nakao e Prof ${ }^{\circ}$. Érica Y. Yoshioka, por serem profissionais dedicados ao ensino e gostar do que faz.

Ao Laboratório de Tecnologia de Pavimento da Escola Politécnica da USP- LPT - POLIUSP, a responsável Prof ${ }^{\circ}$. Dra. Liedi, aos técnicos Edson e Kendi.

Ao Canteiro Experimental - FAU-USP com os responsáveis Prof ${ }^{\circ}$. Dr. Reginaldo Ronconi e Prof ${ }^{\circ}$. Dra. Érica Yoshioka e ao técnico Romerito, nos quais foram dados todos os respaldos necessários para a realização dessa pesquisa.

E por último, mas não menos especial, o meu agradecimento à própria terra... 
"O que chamamos de moderno, pode ser simplesmente o que não merece permanecer para se tornar antigo"

(Dante Alighieri) 


\section{RESUMO}

Este trabalho pretende estabelecer bases para a análise de estruturas correntes feitas em taipa de pilão. Esse material, constituído quase que somente por terra obtida no local da obra, tem todas as propriedades do que se chama uma construção sustentável, incluindo um mínimo de consumo de energia, se comparado as estruturas convencionais em aço e concreto.

O texto é dividido em três temas principais. O primeiro trata de revisão da pouca literatura técnica existente sobre o assunto, incluindo a descrição das técnicas de construção e de seleção e preparo dos materiais. O segundo abrange a pesquisa feita pela autora no preparo e ensaio de corpos de prova do material, com a intenção de obter parâmetros que são o insumo para a análise propriamente dita de estruturas desse tipo. Esta análise é a terceira parte do trabalho, a qual consiste no processamento de modelos tridimensionais de elementos finitos de estruturas de construções hipotéticas em taipa de pilão.

Palavras Chaves: estruturas (análise), taipa de pilão, construção em terra, técnicas tradicionais. 


\begin{abstract}
This work intends to provide basis for the analysis of common structures built of rammed earth. This material, constituted almost entirely of earth obtained in the place where the building will stand, has all the properties of what is known as sustainable construction, including a minimal consume of energy, as compared to conventional steel or concrete structures.

The text is divided in three main themes. The first reports the review the little existing technical literature on the subject, including the description of the building procedures and the selection and preparation of the materials. The second reports research conducted by the author on the preparation and testing of samples of the material, in order to obtain the parameters needed in the analysis of structures of this type. This is the third part of the work, consisting in the numerical analysis of three dimensional finite element models of hypothetical buildings made of rammed earth.
\end{abstract}

Keywords: structure (Analysis), rammed earth, land construction, traditional techniques. 


\section{LISTA DE ABREVIATURAS E SIGLAS}

ABCP: Associação Brasileira de Cimento Portland.

ABCTerra: Associação Brasileira dos Construtores em Terra.

ABNT: Associação Brasileira de Normas Técnicas

CEPED: Centro de Pesquisa e Desenvolvimento da Bahia.

CETEC: Fundação Centro Tecnológico de Minas Gerais.

CMMAD: Comissão Mundial sobre Meio Ambiente e Desenvolvimento.

CONPRESP: Conselho Municipal de Preservação do Patrimônio Histórico, Cultural e Ambiental da Cidade de São Paulo.

CRAterre: Centre Internacional de Recherche et D’Application pour la Construction en terre.

CYTED: Programa Iberoamericano de Ciencia y Tecnologia para el Desarrollo.

DEPEA / BNH: Departamento de Estudos e Pesquisas Aplicadas do Banco

Nacional da Habitação.

FAU-USP: Faculdade de Arquitetura e Urbanismo - Universidade de São Paulo.

FEC: Faculdade de Engenharia Civil.

ICPA: Instituto Del Cemento Portland Argentino.

LPT: Laboratório de tecnologia de Pavimento da Escola Politécnica da USP.

ONU: Organização das Nações Unidas.

PCA: Portland Cement Association.

POLI-USP: Escola Politécnica de São Paulo - Universidade de São Paulo.

TCPO: Tabela de Composições de Preços para Orçamentos.

UNICAMP: Universidade Estadual de Campinas.

UNIMEP: Universidade Metodista de Piracicaba.

USP: Universidade de São Paulo.

UNESP: Universidade Estadual Paulista. 


\section{LISTA DE SÍMBOLOS}

LL: Limite de Liquidez

LP: Limite de Plasticidade

IP: Índice de Plasticidade

$\mathbf{m}$ : unidade de medida em metros.

$\%$ : unidade de medida em porcentagem.

$\mathbf{m}^{3}$ : unidade de medida em volume (metros cúbicos).

$\mathbf{m}^{2}$ : unidade de medida em área (metros quadrados).

mm: unidade de medida em milímetro.

cm: unidade de medida em centímetro.

kg: quilograma

min.: minuto

ph: potencial hidrogeniônico

h: altura

d: espessura da parede

$\boldsymbol{\sigma}$ : tensão normal

L: comprimento

D: diâmetro

P: peso

fc: resistência a compressão

ft: resistência a tração

Pa: Pascal

MPa: mega Pascal

GPa: giga Pascal

kgf: quilograma força

E: módulo de elasticidade

$\gamma:$ peso específico

V: volume

v: coeficiente de Poisson

$\boldsymbol{\varepsilon}$ : deformação

$\mathbf{N}$ : Newton 